ORIGINAL MANUSCRIPT

\title{
CCDC26, CDKN2BAS, RTEL1 and TERT Polymorphisms in pediatric brain tumor susceptibility
}

\author{
Maral Adel Fahmideh*, Catharina Lavebratt ${ }^{1}$, Joachim Schüz ${ }^{2}$, \\ Martin Röösli ${ }^{3,4}$, Tore Tynes ${ }^{5,6}$, Michael A.Grotzer ${ }^{7}$, Christoffer Johansen ${ }^{8,9}$, \\ Claudia E.Kuehni ${ }^{10}$, Birgitta Lannering ${ }^{11}$, Michaela Prochazka, \\ Lisbeth S.Schmidt ${ }^{12}$ and Maria Feychting
}

Unit of Epidemiology, Institute of Environmental Medicine, Karolinska Institutet, Box 210, Stockholm SE-171 77, Sweden, ${ }^{1}$ Neurogenetics Unit, Department of Molecular Medicine and Surgery, Karolinska Institutet, and Center for Molecular Medicine, Karolinska University Hospital, Stockholm SE-171 76, Sweden, ${ }^{2}$ Section of Environment and Radiation, International Agency for Research on Cancer (IARC), 69372 Lyon CEDEX 08, France, ${ }^{3}$ Department of Epidemiology and Public Health, Swiss Tropical and Public Health Institute, Basel 4002, Switzerland, ${ }^{4}$ University of Basel, Basel 4003, Switzerland, ${ }^{5}$ The Cancer Registry of Norway, Oslo N-0304, Norway, ${ }^{6}$ National Institute of Occupational Health, Oslo NO-0033, Norway, ${ }^{7}$ Department of Oncology, University Children's Hospital of Zurich, Zurich 8091, Switzerland, ${ }^{8}$ Unit of Survivorship, The Danish Cancer Society Research Centre, Copenhagen DK-2100, Denmark, ${ }^{9} 5073$ Oncology Clinic, Finsen Centre Rigshospitalet, University of Copenhagen, Copenhagen DK-2100, Denmark, ${ }^{10}$ Swiss Childhood Cancer Registry, Institute of Social and Preventive Medicine, University of Bern, Bern 3012, Switzerland, ${ }^{11}$ Department of Clinical Sciences, Pediatric Oncology, University of Gothenburg, SE 41685 Gothenburg, Sweden and ${ }^{12}$ Department of Pediatric Oncology, University Hospital Rigshospitalet, Copenhagen DK-2100, Denmark

*To whom correspondence should be addressed. Tel. +46 8524839 54; Fax. +46 8 313961; Email: maral.adel.fahmideh@ki.se

\begin{abstract}
The role of genetic polymorphisms in pediatric brain tumor (PBT) etiology is poorly understood. We hypothesized that single nucleotide polymorphisms (SNPs) identified in genome-wide association studies (GWAS) on adult glioma would also be associated with PBT risk. The study is based on the Cefalo study, a population-based multicenter case-control study. Saliva DNA from 245 cases and 489 controls, aged 7-19 years at diagnosis/reference date, was extracted and genotyped for 29 SNPs reported by GWAS to be significantly associated with risk of adult glioma. Data were analyzed using unconditional logistic regression. Stratified analyses were performed for two histological subtypes: astrocytoma alone and the other tumor types combined. The results indicated that four SNPs, CDKN2BAS rs4977756 ( $p=0.036)$, rs1412829 $(p=0.037)$, rs2157719 $(p=0.018)$ and rs1063192 ( $p=0.021)$, were associated with an increased susceptibility to PBTs, whereas the TERT rs2736100 was associated with a decreased risk $(p=0.018)$. Moreover, the stratified analyses showed a decreased risk of astrocytoma associated with RTEL1 rs6089953, rs6010620 and rs2297440 ( $p_{\text {trend }}=0.022, p_{\text {trend }}=0.042, p_{\text {trend }}=0.029$, respectively) as well as an increased risk of this subtype associated with RTEL1 rs4809324 ( $\left.p_{\text {trend }}=0.033\right)$. In addition, SNPs rs 10464870 and rs891835 in CCDC26 were associated with an increased risk of non-astrocytoma tumor subtypes $\left(p_{\text {trend }}=0.009, p_{\text {trend }}=0.007\right.$, respectively). Our findings indicate that SNPs in CDKN2BAS, TERT, RTEL1 and CCDC26 may be associated with the risk of PBTs. Therefore, we suggest that pediatric and adult brain tumors might share common genetic risk factors and similar etiological pathways.
\end{abstract}




\section{Abbreviataions}

\begin{tabular}{ll} 
CI & confidence interval \\
GWAS & genome-wide association studies \\
OR & odds ratio \\
PBT & pediatric brain tumor \\
SNP & single nucleotide polymorphism \\
\hline
\end{tabular}

\section{Introduction}

Brain tumors are the second most common type of pediatric cancer and the leading cause of childhood cancer mortality. The etiology of pediatric brain tumors (PBTs) is poorly understood (1). As in adults (2), the only established risk factors for brain tumors in children are exposure to high doses of ionizing radiation and several inherited disorders, and these cause only a minority of cases. Therefore, it is likely that brain tumorigenesis results from complex interactions between genetic and epigenetic variations in concert with exposure to environmental factors (1).

Although large genetic studies on adult brain tumors have been conducted (3-7), very few and only small studies of brain tumors in children and adolescents have been reported (8-11). In the last few years, four genome-wide association studies (GWAS) on adult glioma identified seven susceptibility loci at 5p15.33 (TERT), 8q24.21 (CCDC26), 9p21.3 (CDKN2A-CDKN2B), 20q13.33 (RTEL1), 11q23.3 (PHLDB1) and 7p11.2 (EGFR) (4-7). However, limited data are available on the role of genetic polymorphisms in the etiology of PBTs, probably because of difficulties in collecting a sufficient number of DNA samples. Considering this lack of knowledge about genetic risk factors for brain tumors in children, it is important to identify germ-line DNA polymorphisms that might influence the susceptibility to PBTs.

The aim of this study, based on the largest series of PBT cases to date, was to test the hypothesis that the single nucleotide polymorphisms (SNPs) identified by GWAS on adult glioma are also associated with the risk of brain tumors in children.

\section{Materials and methods}

\section{Study population and procedures}

This study is based on the Cefalo study, a large, international, populationbased, case-control study of brain tumors in children and adolescents conducted in centers in Sweden, Denmark, Norway and Switzerland. All centers followed a common protocol for data collection, as described in more detail elsewhere $(12,13)$. Eligible cases were children aged 7-19 years during the period 1 April 2004 to 31 August 2008, diagnosed with a primary intracranial brain tumor defined according to the International Classification of Childhood Cancer, third edition (ICCC-3) (14), group III, restricted to the third edition of the International Classification of Diseases for Oncology (ICD-O-3), location C71 and subclassified according to the fourth edition of the World Health Organization (WHO) classification of tumors of the central nervous system (15). Medulloblastoma cases will be the subject of a separate study and therefore have been excluded from the present analysis. Two controls per case were randomly selected from the general population matched to the case by age, sex and geographical region. Interviews were conducted with 352 (82\%) cases and 646 (71\%) controls. Participants with neurofibromatosis or tuberous sclerosis were excluded from the analyses. The study was approved by the national data protection boards and ethical committees in all participating countries, and written informed consent was obtained from all participants and/or their parents.

The Oragene self-collection kit (DNA Genotek, Ottawa, ON, Canada) was used for saliva collection and DNA extraction, following the manufacturer's recommended protocol. DNA samples are stored at the Karolinska Institutet Biobank. The DNA yield was quantitated by using PicoGreen
(Invitrogen, Carlsbad, CA). Overall, saliva DNA from 245 cases and 489 controls was included in this study.

\section{SNP selection and genotyping}

A total of 29 SNPs reported by GWAS to be significantly associated with risk of adult glioma were selected for genotyping (4-7). Genotyping was performed at the Mutation Analysis core Facility, Clinical Research Centre, Huddinge University Hospital, Stockholm, Sweden, with staff blinded to sample status, using the Sequenom iPlex Gold platform with matrixassisted laser desorption/ionization-time-of-flight (MALDI-TOF) mass spectrometry. The average success rate was $97 \%$ and the concordance rate for duplicate genotyping was $100 \%$.

\section{Statistical analysis}

The consistency of allele frequencies with Hardy-Weinberg equilibrium was assessed in the controls for all SNPs using the $\chi^{2}$ goodness-of-fit test, and $p<0.001$ was considered statistically significant. Analyses were performed based on the subset of Cefalo subjects who provided saliva sample. Unconditional logistic regression was used to estimate the association between SNPs and PBT susceptibility based on the Cochran-Armitage trend test of additivity (trend) as well as dominant (DOM) and recessive (REC) models, with adjustment for the matching variables (age, sex and country). The allelic frequencies of the genotyped SNPs were compared between cases and controls using the $\chi^{2}$ test. Analyses were also conducted stratified by astrocytoma alone and the combination of other tumor types, including ependymoma, intracranial embryonal tumors (except medulloblastoma), other gliomas, other specified intracranial neoplasms and unspecified intracranial neoplasms. Country specific analyses were performed to assess consistency across countries. The Wald test was used to evaluate the significance of interactions between SNPs and demographic variables and $\mathrm{D}^{\prime}$, a measure of the linkage disequilibrium (LD) between the genotyped SNPs, was calculated. Haploblocks were defined based on the default linkage disequilibrium block parameters in Haploview v4.2. Haplotype analyses were performed for the haplotype blocks harboring the SNPs that were found to be associated with PBTs. Haplotypes with a frequency of $>1 \%$ were considered in the analyses. The effects of specific haplotypes were analyzed if the distribution of all the haplotypes was suggestively different between cases and controls ( $p<0.05$ for all PBTs; $p<0.1$ for subgroup analyses). Selection of SNPs for the analyses was based on a priori knowledge from GWAS on adults, and therefore odds ratios (ORs) are presented with $95 \%$ confidence intervals (CIs). The possibility of falsepositive findings was, however, considered by also providing the reference $p$ value for an experiment-wide significance with Bonferroni correction. The analyses were performed using PLINK v1.07 (16) and SAS statistical software version 9.3 (SAS Institute, Cary, NC).

\section{Results}

We successfully genotyped 29 SNPs in 245 cases and 489 controls. The distributions of allele frequencies in the controls were in agreement with the Hardy-Weinberg equilibrium. Table 1 shows demographic characteristics of cases and controls and the distributions of diagnostic subtypes. The age and sex distributions were similar in cases and controls. More than $50 \%$ of cases were diagnosed with astrocytoma. No significant interactions were detected between SNPs and confounders including age, sex and country (Table 2).

As shown in Table 2, TERT rs2736100 A allele was associated with a decreased risk of PBTs $\left[\mathrm{OR}_{\mathrm{DOM}} 0.66\right.$ (95\% CI $0.46-0.93$ ), $p=0.018]$, whereas the SNPs rs $4977756 \mathrm{G}$ allele $\left[\mathrm{OR}_{\mathrm{DoM}} 1.45(95 \%\right.$ CI 1.03-2.06), $p=0.036$ ], rs $1412829 \mathrm{G}$ allele [OR $_{\text {DoM }} 1.45$ (95\% CI $1.02-2.05), p=0.037$ ], rs2157719 C allele [OR $\mathrm{Dom}_{1.53} 195 \% \mathrm{CI} 1.08-$ 2.19), $p=0.018$ ] and rs1063192 G allele [OR DoM $_{1.53}$ (95\% CI 1.072.19), $p=0.021$ ] in CDKN2BAS were associated with increased susceptibility to this tumors.

The stratified analyses of two histological subtypes indicated that the risk effects of CDKN2BAS rs1063192, rs2157719, rs1412829 and rs4977756 remained significant in patients with astrocytoma 
Table 1. Characteristics of cases and controls

\begin{tabular}{|c|c|c|}
\hline Characteristics & Cases & Controls \\
\hline No. of participants & 245 & 489 \\
\hline \multicolumn{3}{|l|}{ Sex } \\
\hline Males & $136(56 \%)$ & $261(53 \%)$ \\
\hline Females & $109(44 \%)$ & $228(47 \%)$ \\
\hline \multicolumn{3}{|l|}{ Age-group (at reference date) } \\
\hline 7-9 years old & $48(20 \%)$ & $112(23 \%)$ \\
\hline 10-14 years old & $108(44 \%)$ & $219(45 \%)$ \\
\hline 15-19 years old & $89(36 \%)$ & $158(32 \%)$ \\
\hline \multicolumn{3}{|l|}{ Country } \\
\hline Sweden & $106(43 \%)$ & $174(36 \%)$ \\
\hline Norway & $24(10 \%)$ & $62(13 \%)$ \\
\hline Denmark & $62(25 \%)$ & $134(27 \%)$ \\
\hline Switzerland & $53(22 \%)$ & $119(24 \%)$ \\
\hline \multicolumn{3}{|l|}{$\begin{array}{l}\text { Type of tumor (International Classification } \\
\text { of Childhood Cancer-3 group III) }\end{array}$} \\
\hline Astrocytoma (IIIb) & $134(55 \%)$ & \\
\hline Pilocytic astrocytoma & 93 & \\
\hline Supependymal giant cell astrocytoma & 5 & \\
\hline Pleomorphic xanthoastrocytoma & 4 & \\
\hline Diffuse astrocytoma & 13 & \\
\hline Anaplastic astrocytoma & 11 & \\
\hline Fibrillary astrocytoma & 2 & \\
\hline Glioblastoma & 5 & \\
\hline Giant cell glioblastoma & 1 & \\
\hline Other gliomas (IIId) & $20(8 \%)$ & \\
\hline Malignant glioma & 11 & \\
\hline Oligoastrocytoma & 2 & \\
\hline Oligodendroglioma & 6 & \\
\hline Anaplastic oligodendroglioma & 1 & \\
\hline Ependymoma (IIIa) & $19(8 \%)$ & \\
\hline Subependymoma & 2 & \\
\hline Choroid plexus papilloma & 4 & \\
\hline Choroid plexus caricinoma & 1 & \\
\hline Ependymoma & 7 & \\
\hline Papillary ependymoma & 1 & \\
\hline Anaplastic ependymoma & 4 & \\
\hline Intracranial embryonal tumors (IIIc) & $7(3 \%)$ & \\
\hline CNS primitive neuroectodermal & 6 & \\
\hline \multicolumn{3}{|l|}{ tumor } \\
\hline Neuroepithelioma & 1 & \\
\hline $\begin{array}{l}\text { Other specified intracranial neoplasms } \\
\text { (IIIe) }\end{array}$ & $49(20 \%)$ & \\
\hline Germinoma & 7 & \\
\hline Yolk sac tumor & 1 & \\
\hline Teratoma, mature & 1 & \\
\hline Haemangioblastoma & 1 & \\
\hline Desmoplastic infantile ganglioglioma & 2 & \\
\hline $\begin{array}{l}\text { Dysembryoplastic neuroepithelial } \\
\text { tumor }\end{array}$ & 6 & \\
\hline Ganglioglioma & 26 & \\
\hline Anaplastic ganglioglioma & 1 & \\
\hline Centrol neurocytoma & 3 & \\
\hline Neurilemoma & 1 & \\
\hline Unspecified intracranial neoplasm (IIIf) & $16(6 \%)$ & \\
\hline
\end{tabular}

${ }^{\text {a}}$ Restricted to ICD-O-3 location C71, subclassified according to World Health Organization histological subclassification, 2007; patients with neurofibromatosis and tuberous sclerosis were excluded.

$\left(p_{\text {trend }}=0.036, p_{\text {trend }}=0.034, p_{\text {trend }}=0.044\right.$ and $p_{\text {trend }}=0.023$, respectively), whereas the protective effect of TERT rs2736100 was more evident in patients with other brain tumor subtypes $\left[\mathrm{OR}_{\mathrm{DOM}} 0.53\right.$ (95\% CI 0.34-0.85), $p=0.007]$. Moreover, the stratified analyses showed a decreased risk of astrocytoma associated with the polymorphisms RTEL1 rs6089953 A allele, rs6010620 A allele and rs2297440 T allele $\left(p_{\text {trend }}=0.022, p_{\text {trend }}=0.042\right.$ and $p_{\text {trend }}=0.029$, respectively), as well as an increased risk of this subtype associated with the C allele of RTEL1 rs4809324 ( $p_{\text {trend }}=0.033$ ). In addition, an increased risk of non-astrocytoma tumor subtypes was associated with the SNPs rs $10464870 \mathrm{C}$ allele and rs891835 G allele in CCDC26 $\left(p_{\text {trend }}=0.009\right.$ and $p_{\text {trend }}=0.007$, respectively) (Table 3).

The non-significant findings, possibly resulting from the limited statistical power of the study, are shown in the online appendix Supplementary Tables 1-3 (available at Carcinogenesis Online) and the raw data showing the number of cases and controls for each genotype of significant SNPs are reported in Supplementary Table 4 (available at Carcinogenesis Online).

Strong linkage disequilibrium $\left(D^{\prime} \geq 0.95\right)$ was observed between three of the genotyped SNPs in CDKN2BAS (rs1412829, rs2157719 and rs1063192) and four SNPs in RTEL1 (rs6089953, rs6010620, rs2297440 and rs4809324). For each of the two blocks, three haplotypes with frequency of $>1 \%$ were found. The distribution of haplotypes was different for PBT patients compared with controls for the CDKN2BAS block $\left(\chi^{2}=7.0, \mathrm{df}=2\right.$ and $p=0.030)$ and showed a tendency to be different for the RTEL1 block $\left(\chi^{2}=5.9, \mathrm{df}=2\right.$ and $\left.p=0.053\right)$. The most common haplotype (ATA) of CDKN2BAS SNPs had a significant protective effect compared with the other haplotypes combined [OR 0.75 (95\% CI $0.60-0.93), p=0.009$ ], whereas the second most common haplotype (GCG) had a significant risk effect [OR 1.32 (95\% CI 1.06-1.64), $p=0.012]$. The haplotype analyses suggested an increased risk of PBTs by increasing the number of risk alleles in CDKN2BAS and RTEL1 SNPs. In the astrocytoma subgroup, the same haploblocks and haplotypes with frequencies of $>1 \%$ were detected. However, in this subgroup, the distribution of haplotypes in the RTEL1 block was significantly different between patients and controls $\left(\chi^{2}=9.0, \mathrm{df}=2\right.$ and $\left.p=0.011\right)$, whereas this difference was not statistically significant in the CDKN2BAS block $\left(\chi^{2}=5.7\right.$, $\mathrm{df}=2$ and $p=0.059)$. In the astrocytoma subgroup, a significant protective effect was observed for the most common haplotype (ATA) of CDKN2BAS SNPs compared with the other haplotypes combined [OR 0.73 (95\% CI 0.56-0.95), $p=0.021$ ], whereas the second most common haplotype (GCG) showed a significant risk effect [OR 1.34 (95\% CI 1.02-1.76), $p=0.036$ ]. Moreover, in the RTEL1 block, the second most common haplotype (AATT) had a significant protective effect compared with the other haplotypes combined [OR 0.67 (95\% CI 0.47-0.95), $p=0.023$ ], whereas the third most common haplotype (GGCC) had a significant risk effect [OR 1.57 (95\% CI 1.06-2.34), $p=0.026$ ] (Table 4).

Overall, we performed 116 testing procedures as described above. When the Bonferroni correction is applied, the reference $p$ value is 0.0004 for an experiment-wide significance level of 0.05 , and 0.0009 for a significance level of 0.10 ; none of the observed associations met these limits. The consistency of results across countries was investigated and the results of stratified analyses are reported in the online appendix Supplementary Tables 5-8 (available at Carcinogenesis Online). No significant differences between countries were observed.

\section{Discussion}

The results of this study indicate that several SNPs associated with adult glioma risk are also associated with the risk of PBTs. Our findings suggest that SNPs rs4977756 G allele, rs1412829 G allele, rs2157719 C allele and rs1063192 G allele in CDKN2BAS may increase the risk of PBTs, whereas the A allele of TERT rs2736100 polymorphism may confer protection against PBTs. In addition, polymorphisms rs6089953 A allele, rs6010620 A allele 


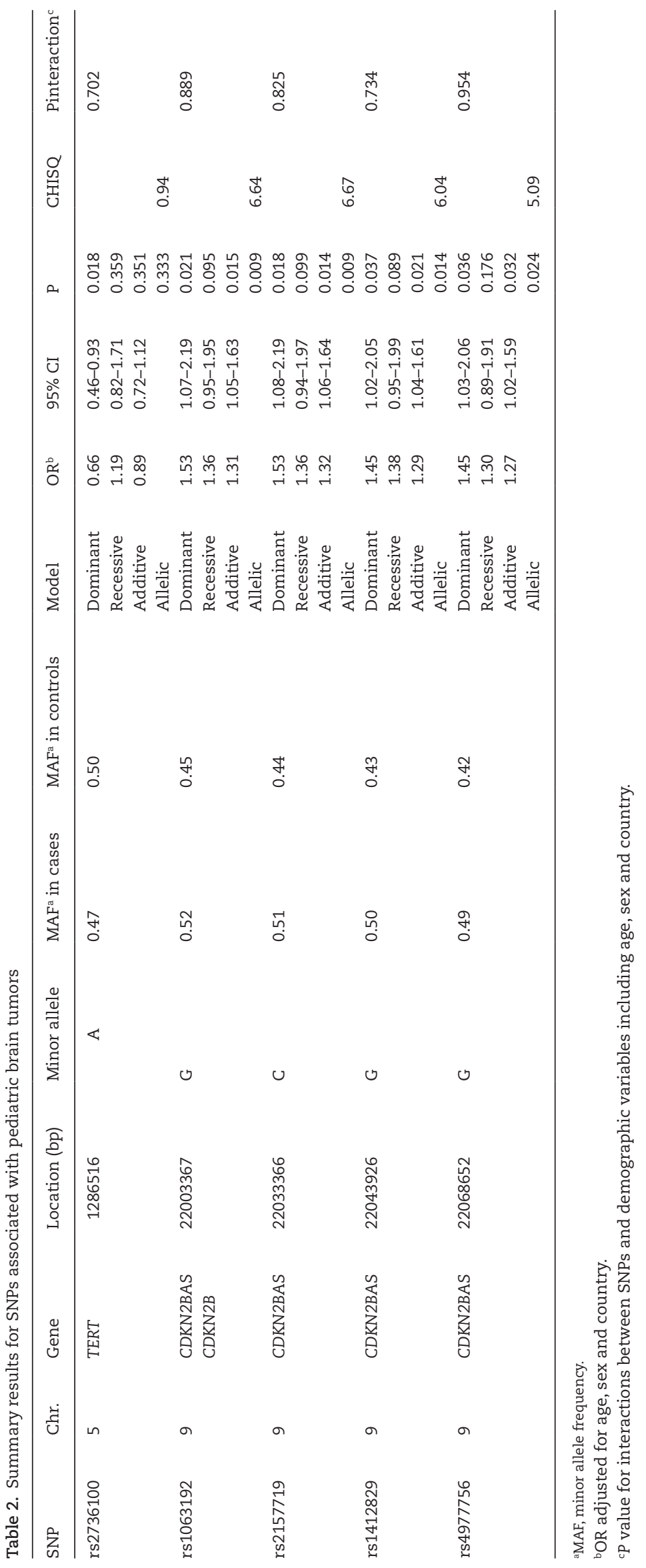


880 | Carcinogenesis, 2015, Vol. 36, No. 8

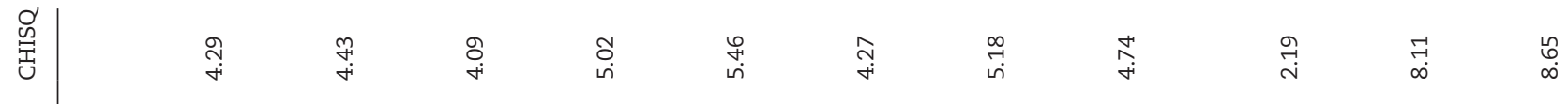

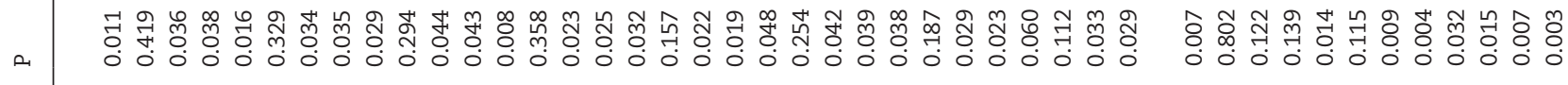

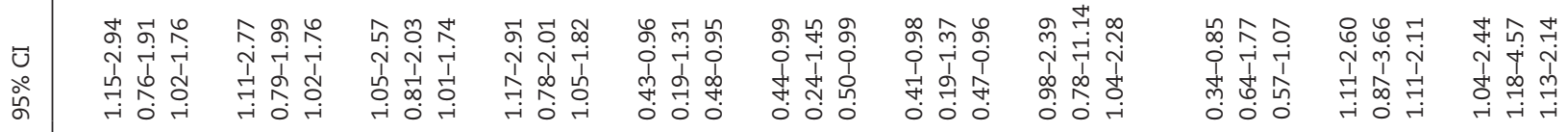

番 $\quad$ 茫

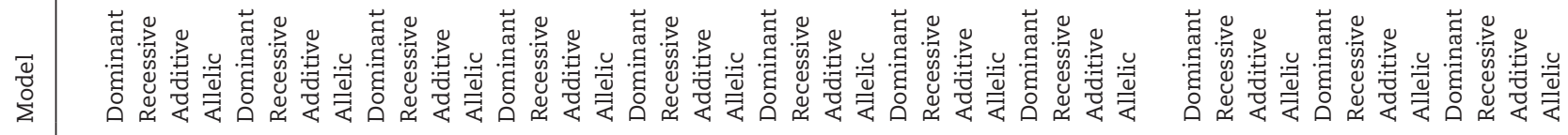

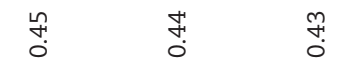

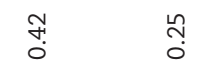

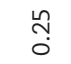

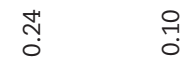

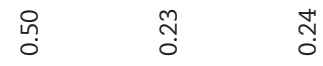

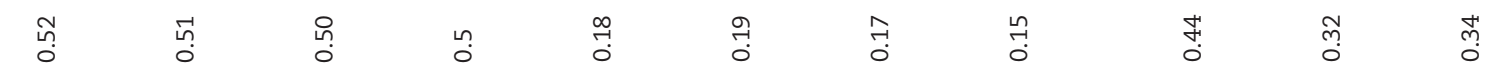

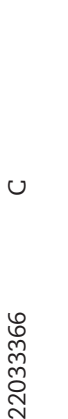

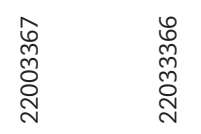

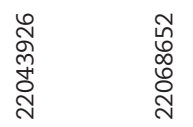

$\infty$
ठิ
तิ

ले
Oे
ָे

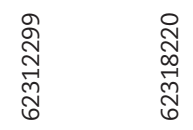

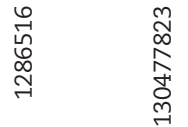

กิ
†ิ
के
감

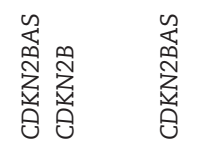

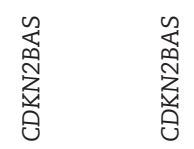

㞼

茞

䓌䓌

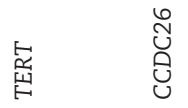

đั

$a$

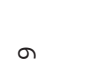

ㅇำ

ำ

ำ

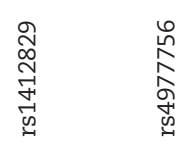

$n$
గू
Oे
0
0
0
0

웡
0
0
0
0
0
0

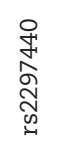

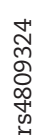



0
$\substack{\infty \\ 0 \\ 0 \\ 0 \\ 0 \\ 0 \\ 0 \\ 0}$ 
Table 4. Haplotype analysis of SNPs in CDKN2BAS and RTEL1

\begin{tabular}{|c|c|c|c|c|c|}
\hline SNPs & Haplotype & Frequency & $\mathrm{OR}^{\mathrm{a}}$ & $95 \%$ CI & $P$ \\
\hline \multirow[t]{3}{*}{ CDKN2BAS: rs1412829, rs2157719, rs1063192 } & ATA & 0.52 & 0.75 & $0.60-0.93$ & 0.009 \\
\hline & GCG & 0.45 & 1.32 & $1.06-1.64$ & 0.012 \\
\hline & ATG & 0.02 & 0.89 & $0.56-1.43$ & 0.814 \\
\hline \multirow[t]{3}{*}{ RTEL1: rs6089953, rs6010620, rs2297440, rs4809324 } & GGCT & 0.65 & 1.03 & $0.81-1.32$ & 0.787 \\
\hline & AATT & 0.23 & 0.79 & $0.61-1.02$ & 0.074 \\
\hline & GGCC & 0.11 & 1.39 & $0.99-1.95$ & 0.055 \\
\hline \multicolumn{6}{|l|}{ Astrocytoma } \\
\hline \multirow[t]{3}{*}{ CDKN2BAS: rs1412829, rs2157719, rs1063192 } & ATA & 0.53 & 0.73 & $0.56-0.95$ & 0.021 \\
\hline & GCG & 0.44 & 1.34 & $1.02-1.76$ & 0.036 \\
\hline & ATG & 0.02 & 0.79 & $0.21-2.94$ & 0.725 \\
\hline \multirow[t]{3}{*}{ RTEL1: rs6089953, rs6010620, rs2297440, rs4809324 } & GGCT & 0.65 & 1.06 & $0.81-1.39$ & 0.676 \\
\hline & AATT & 0.23 & 0.67 & $0.47-0.95$ & 0.023 \\
\hline & GGCC & 0.11 & 1.57 & $1.06-2.34$ & 0.026 \\
\hline
\end{tabular}

aORs for haplotype compared with all other haplotypes adjusted for age, sex and country.

and rs2297440 T allele in RTEL1 were associated with decreased susceptibility to astrocytoma, whereas the C allele of RTEL1 rs4809324 was associated with an increased risk of this subtype. Furthermore, an increased risk of non-astrocytoma tumor subtypes associated with polymorphisms CCDC26 rs10464870 C allele and rs891835 G allele was detected. Our findings suggest that genetic risk profiles of PBTs differ by histology.

To our knowledge, this study represents the largest series of pediatric brain tumor cases assembled for genetic association testing to date. The association between the 29 SNPs investigated in this study and the risk of PBTs has not been examined in previous studies (8-11). The SNPs were selected a priori for analyses in this study based on findings in GWAS on adult glioma, and our significant findings of associations between SNPs in CDKN2BAS, TERT, RTEL1 and CCDC26, and risk of PBTs were consistent with findings on adult brain tumors with respect to the direction of ORs for the minor alleles (4-7).

CDKN2BAS (ANRIL) encodes antisense non-coding RNA in the INK4 locus which is a long non-coding RNA (ncRNA). The exact function of CDKN2BAS is unclear, but it is known to be involved in regulating the expression of CDKN2A and CDKN2B genes that encode cyclin-dependent kinase inhibitors and block cell cycle division during the G1/S phase. Therefore, CDKN2BAS has a regulatory role in the context of cellular proliferation, and its alterations result in abnormal self-renewing capabilities typical of cancer cells $(17,18)$. Germ-line mutations in CDKN2BAS predispose to a wide variety of human cancers $(19,20)$.

Telomerase reverse transcriptase (TERT) is a catalytic subunit of telomerase that maintains telomere by adding telomeric repeat sequences onto chromosome ends. Telomerase expression can prevent telomere erosion in most eukaryotic cells, and cancer cells can prevent telomere loss through the abnormal upregulation of telomerase (21). The mutant allele of TERT rs2736100, which is an intronic polymorphism, may downregulate telomerase expression and consequently decrease the risk of brain tumors.

RTEL1 produces regulator of telomere elongation helicase 1 which is essential for telomere maintenance and genome stability by preventing homologous recombination (22). Polymorphisms in RTEL1 are correlated with high grade glioma in adults $(4,5,7,23)$. In contrast, CCDC26 variations are associated with low grade tumors $(4,5,23)$. CCDC26 encodes a retinoic aciddependent regulator of cell differentiation and death. CCDC26 increases apoptosis induced by death stimuli in neuroblastoma cells (24) and in glioblastoma cells with downregulation of telomerase activity (25).
The majority of genetic variations found in this study to be associated with the risk of PBTs are related to telomerase activity which has an important role in the initiation and progression of gliomas (26). Moreover, it has been shown that telomerase expression is related to high grade gliomas and poor survival $(27,28)$. Thus, telomerase could be considered as a therapeutic target for brain tumors $(29,30)$.

The aim of this study was to provide evidence of the associations between SNPs and PBT risk, and not to investigate the mechanisms behind such associations; nevertheless the fact that we did not consider the effect of environmental risk factors represents a limitation of this work. Therefore, additional studies are needed to examine potentially relevant gene-environment interactions and to explore the mechanisms through which these genetic polymorphisms influence cancer susceptibility.

The present study was conducted based on a specific hypothesis that may lead to detection of clinically meaningful risk and protective factors. Moreover, the selection of SNPs for analysis was based on a priori knowledge from GWAS on adults, and therefore Bonferroni correction may be overly conservative and may make researchers miss important findings (31). To be able to evaluate potential false-positive findings, reference $p$ values with Bonferroni corrections have been presented and the consistency of results across four countries has been reported. No significant differences between countries were observed. Replication studies are necessary to confirm these results in larger sample sizes.

In conclusion, the present findings indicate that SNPs in CDKN2BAS are associated with increased susceptibility to PBTs, whereas TERT polymorphisms may decrease the risk of these tumors. Moreover, polymorphisms in RTEL1 and CCDC26 genes are associated with the risk of astrocytoma and non-astrocytoma subtypes, respectively. Thus, we suggest that pediatric and adult brain tumors might share common genetic risk factors and similar etiological pathways.

\section{Supplementary material}

Supplementary Tables 1-8 can be found at http://carcin.oxfordjournals.org/.

\section{Funding}

Swedish Council for Working Life and Social Research (20040504 and 2007-0224); Swedish Research Council (K200870X-15366-04-3); Swedish Cancer Society (09 0666); Swedish 
Childhood Cancer Society (PROJ06/050 and PROJ09/086); Swedish Radiation Protection Authority (SSI P 1572); Danish Strategic Research Council (2103-05-0006 and 2064-04-0010); Swiss Federal Office of Public Health (05.001626); Swiss Research Foundation on Mobile Communication (A2006.18); Swiss National Science Foundation (PDFMP3_122873); Research Council of Norway (175163/V40).

\section{Acknowledgements}

We gratefully acknowledge collaboration with and support from clinicians and other hospital staff in all countries, as well as assistance from national and local cancer registers with identification of patients. We also acknowledge the skillful work of the research nurses, interviewers, and research assistants in all countries. Finally, we acknowledge all those with whom we have collaborated previously within the CEFALO study. Please see Aydin et al. for an exhaustive list (12). Conflict of Interest Statement: None declared.

URLs: PLINK: http://pngu.mgh.harvard.edu/ purcell/plink/; SAS: http://www.sas.com/

\section{References}

1. Pollack, I.F. et al. (2011) Childhood brain tumors: epidemiology, current management and future directions. Nat. Rev. Neurol., 7, 495-506.

2. Fisher, J.L. et al. (2007) Epidemiology of brain tumors. Neurol. Clin., 25, 867-890.

3. Adel Fahmideh, M. et al. (2014) Association between DNA repair gene polymorphisms and risk of glioma: a systematic review and metaanalysis. Neuro Oncol.,16, 807-814.

4. Rajaraman, P. et al. (2012) Genome-wide association study of glioma and meta-analysis. Hum. Genet., 131, 1877-1888.

5. Sanson, M. et al. (2011) Chromosome 7p11.2 (EGFR) variation influences glioma risk. Hum. Mol. Genet., 20, 2897-2904.

6. Shete, S. et al. (2009) Genome-wide association study identifies five susceptibility loci for glioma. Nat. Genet., 41, 899-904.

7. Wrensch, M. et al. (2009) Variants in the CDKN2B and RTEL1 regions are associated with high-grade glioma susceptibility. Nat. Genet., 41, 905-908.

8. Jeon, S. et al. (2013) Genetic variants of AICDA/CASP14 associated with childhood brain tumor. Genet. Mol. Res., 12, 2024-2031.

9. Salnikova, L.E. et al. (2010) Association study of xenobiotic detoxication and repair genes with malignant brain tumors in children. Acta Naturae, 2, 58-65.

10. Searles Nielsen, S. et al. (2010) Childhood brain tumors, residential insecticide exposure, and pesticide metabolism genes. Environ. Health Perspect., 118, 144-149.
11. Sirachainan, N. et al. (2008) Folate pathway genetic polymorphisms and susceptibility of central nervous system tumors in Thai children. Cancer Detect. Prev., 32, 72-78.

12. Aydin, D. et al. (2011) Mobile phone use and brain tumors in children and adolescents: a multicenter case-control study. J. Natl Cancer Inst., 103, 1264-1276.

13. Christensen, J.S. et al. (2012) Brain tumors in children and adolescents and exposure to animals and farm life: a multicenter case-control study (CEFALO). Cancer Causes Control, 23, 1463-1473.

14. Steliarova-Foucher, E. et al. (2005) International Classification of Childhood Cancer, third edition. Cancer, 103, 1457-1467.

15. Louis, D.N. et al. (2007) The 2007 WHO classification of tumours of the central nervous system. Acta Neuropathol., 114, 97-109.

16. Purcell, S, et al. (2007) PLINK: a tool set for whole-genome association and population-based linkage analyses. Am J Hum Genet., 81, 559-575.

17. Cunnington, M.S. et al. (2010) Chromosome 9p21 SNPs Associated with Multiple Disease Phenotypes Correlate with ANRIL Expression. PLoS Genet., 6, e1000899.

18. Wapinski, O. et al. (2011) Long noncoding RNAs and human disease. Trends Cell Biol., 21, 354-361.

19. Gu, F. et al. (2013) Common genetic variants in the 9p21 region and their associations with multiple tumours. Br. J. Cancer., 108, 1378-1386.

20. Turnbull, C. et al. (2010) Genome-wide association study identifies five new breast cancer susceptibility loci. Nat Genet., 42, 504-507.

21. Baird, D.M. (2010) Variation at the TERT locus and predisposition for cancer. Expert Rev. Mol. Med., 12, e16.

22. Barber, L.J. et al. (2008) RTEL1 maintains genomic stability by suppressing homologous recombination. Cell, 135, 261-271.

23. Simon, M. et al. (2010) Genetic risk profiles identify different molecular etiologies for glioma. Clin. Cancer Res., 16, 5252-5259.

24. Jiang, M. et al. (2008) Retinoic acid induces caspase-8 transcription via phospho-CREB and increases apoptotic responses to death stimuli in neuroblastoma cells. Biochim. Biophys. Acta., 1783, 1055-1067.

25. Das, A. et al. (2007) Differentiation decreased telomerase activity in rat glioblastoma C6 cells and increased sensitivity to IFN-gamma and taxol for apoptosis. Neurochem. Res., 32, 2167-2183.

26. Shervington, A. et al. (2006) Glioma: what is the role of c-Myc, hsp90 and telomerase? Mol. Cell. Biochem., 283, 1-9.

27. Tabori, U. et al. (2006) Human telomere reverse transcriptase expression predicts progression and survival in pediatric intracranial ependymoma. J. Clin. Oncol., 24, 1522-1528.

28. Tchirkov, A. et al. (2003) Clinical implications of quantitative realtime RT-PCR analysis of hTERT gene expression in human gliomas. $\mathrm{Br}$. J. Cancer, 88, 516-520.

29. Kim, J.H. et al. (2013) Ependymoma in children: molecular considerations and therapeutic insights. Clin. Transl. Oncol., 15, 759-765.

30. Wong, V.C. et al. (2010) Telomerase inhibition as a novel therapy for pediatric ependymoma. Brain Pathol., 20, 780-786.

31. Rothman, K.J. (1990) No adjustments are needed for multiple comparisons. Epidemiology, 1, 43-46. 